\title{
Risk assessment for hereditary breast cancer: BRCA1 and BRCA2
}

by Kendra-Ann I. Seenandan-Sookdeo and Jo-Ann V. Sawatzky

\section{Abstract}

The recent explosion of hereditary breast cancer genetic research has strengthened our understanding of genetic influences on health and disease. These scientific advances have significant implications for clinical oncology nurses and advanced practice nurses. In this paper, the Human Response to Illness Model (Mitchell, Gallucci, \& Fought, 1991) is utilized to gain a comprehensive understanding of breast cancer related to the human response of genetic mutations. Accordingly, the research literature related to the physiological, pathophysiological, behavioural, and experiential perspectives of BRCA-related breast cancer will be highlighted. This research-based evidence will provide oncology nurses with the skills to establish a holistic plan of care for women with an actual or potential genetic risk for breast cancer.

\section{Risk assessment for hereditary breast cancer: BRCA1 and BRCA2}

Breast cancer has touched the lives of many individuals and families worldwide. In Canada, breast cancer is the most frequently diagnosed cancer in women, with the second highest female mortality rate after lung cancer. In 2009, it is estimated that 22,900 Canadian women will receive a positive breast cancer diagnosis, reflecting 5,400 deaths (Canadian Cancer Society, 2009). A strong family history of breast cancer increases an individual's risk profile for the disease. In addition, a positive breast cancer gene mutation will further influence risk profiles. Hereditary breast cancer accounts for $5 \%$ to $10 \%$ of all breast cancer diagnoses, with approximately $3 \%$ to $7 \%$ of this group having a positive BRCA1 or BRCA2 gene mutation (Carroll et al., 2008). Carriers of these genes have a $50 \%$ to $87 \%$ chance of developing breast cancer by the age of 70 (Berliner \& Fay, 2007).

This article utilizes the Human Response to Illness (HRTI) Model (Mitchell et al., 1991) to guide an organized review of the human response of potential or actual hereditary breast cancer related to BRCA genes, by presenting the four interacting perspectives involved in the complex human response to illness. These four domains include physiologic, pathophysiologic, behavioural, and experiential perspectives. Research-based evidence will be utilized to establish a comprehensive understanding of hereditary breast cancer, risk assessment tools, psychological issues, and implications for oncology nurses. Thus, this review will provide clinical nurses with the knowledge needed to initiate evidence-based breast history assessment, client education, supportive counselling and suitable genetic referrals.

\section{Physiologic perspective}

According to Mitchell et al. (1991), the principles of physiologic regulatory responses are derived from an understanding of normal biological functioning of the body. Hence, a discussion of hereditary breast cancer must begin with the fundamentals of cell biology and the role of BRCA1 and BRCA2 in the complex activities that occur within the nucleus of a cell (Loescher \& Whitesell, 2006). The literature provides substantive evidence that cancer is a genetic disease ensuing from gene mutations. Any mutation that causes an increase in the rate of cell division, or a decrease in the normal rate at which cells die, can contribute to the uncontrolled growth of cancerous cells (Loescher et al., 2006). The body's somatic cells (i.e., all human body cells, with the exception of sex cells) contain a nucleus consisting of chromosomes that enclose compact functional units of inheritance, known as genes. Genes are composed of sequences of deoxyribonucleic acid (DNA). DNA encodes for approximately 30,000 genes found in each of the 23 paired human chromosomes (Loescher et al., 2006). Of the 23 paired chromosomes, one chromosome of each pair is contributed to an offspring from the maternal and paternal lineage. Therefore, an offspring has a $50 \%$ chance of inheriting an autosomal dominant gene mutation.
While there are two categories of genes that play a pivotal role in cellular control (i.e., tumour suppressor genes and proto-oncogenes), this review will focus on the two tumour suppressor genes: BRCA1 and BRCA2. These genes play a role in the transcription of DNA and regulate cellular activities by inhibiting growth promoting proteins in order to enable damaged cells to be repaired (Yoshida \& Miki, 2004). Both genes also participate in controlling signals for cell apoptosis or cell death (Loescher et al., 2006). Narod and Foulkes (2004) emphasize that the human genome is preserved by the involvement of BRCA proteins to varying degrees in numerous points of the cell cycle including, but not limited to: cell cycle checkpoints, ubiquitylation, chromatin remodelling, and homologous recombination. These specific sequences of events are innate in mitosis cell division. Checkpoints ensure a cell is prepared for complete cellular division before proceeding to the next phase. Ubiquitylation is a cell cycle process by which proteins are marked for degradation, while chromatin remodelling ensures that breaks in double strand DNA are repaired, and homologous recombination is the arrangement of double strand DNA copying (Narod et al., 2004). Thus, BRCA proteins play a critical role, as gatekeepers in maintaining a number of cellular mechanisms.

\section{Pathophysiologic perspective}

Mitchell et al. (1991) suggest that pathophysiologic responses result in biological decompensation. Therefore, an alteration in a BRCA gene would place an individual at increased risk for ill health. A deleterious mutation in either BRCA gene increases an individual's susceptibility to breast and ovarian cancer, as well as other types of cancers (Berliner et al., 2007). While the majority of breast cancers are sporadic and result from somatic mutations, hereditary cancer susceptibility is related to germline mutations (Fackenthal \& Olopade, 2007).

Chapman (2007) defines germline mutations as an inherited genetic alterations found in the egg or sperm, which are passed on to an offspring. A literature review by Narod et al. (2004) continues to support the two hit model theory regarding hereditary breast cancer. This the-

\section{About the authors}

Kendra-Ann I. Seenandan-Sookdeo, BN, RN, Graduate Student, Research Nurse, CancerCare Manitoba/Manitoba Institute of Cell Biology-Breast Tumour Bank, Winnipeg Regional Health Authority/Health Science Centre-Hereditary Breast \& Ovarian Cancer Clinic, Breast Clinic Nurse, Winnipeg Regional Health Authority-Breast Health Centre. E-mail:

KendraAnn.Seenandan@cancercare.mb.ca

Jo-Ann V. Sawatzky, RN, PhD, Associate Professor, Faculty of Nursing, University of Manitoba.

The authors gratefully acknowledge the following for their review of an earlier draft of this article: Dr Leigh Murphy, Acting Director and Senior Scientist of CancerCare Manitoba Institute of Cell Biology, and Kim Serfas, Genetic Counsellor, Winnipeg Regional Health Authority: Health Science Centre/Hereditary Breast and Ovarian Clinic.

Supported by the following award to the first author: A student scholarship from the Canadian Health Services Research Foundation/Canadian Institutes of Health Research Development of Evidence-based Nursing Practice in Cancer Care, Palliative Care and Cancer Prevention Chair Award held by Dr. Lesley Degner, Faculty of Nursing, University of Manitoba.

"This article is dedicated to my grandmother, Winnifred Ramsay." -Kendra-Ann I. Seenandan-Sookdeo 
ory maintains that cancer can result when an individual who has inherited a BRCA gene mutation from one parent later undergoes somatic mutation in the BRCA gene inherited from the other parent. The clinical significance of one-third of these mutations is unspecified. Consequently, some may be normal variants without pathological consequences because the mutation still results in a functional protein, while others may predispose the individual to cancer susceptibility because of the resulting non-functional protein (Loescher et al., 2006).

Defective BRCA genes have potential negative implications in the transcription of DNA, cell cycle regulation, DNA repair, checkpoint controls, ubiquitylation, and chromatin remodelling. According to Yoshida et al. (2004), altered BRCA proteins can interfere with critical protein connections. Therefore, changes in BRCA genes can contribute to tumour development, by a process known as tumourigenesis (Yoshida et al., 2004).

\section{Behavioural perspective}

Behavioural responses in the HRTI Model are described as observable and measurable behaviours, which may be indicative of an underlying sign or symptom (Mitchell et al., 1991). The model also proposes that environmental, as well as non-modifiable (i.e., genetic makeup) and modifiable (i.e., lifestyle) factors interact with the four perspectives of human response to illness to influence an individual's health response. Risk assessment tools measure an individual's underlying potential of a non-modifiable inherited risk for a BRCA mutation (Mitchell et al., 1991). In the primary care setting, the key measurement tool is a comprehensive genetic oncology health history assessment, which takes into account personal and family health issues in the appraisal of eligibility for genetic counselling (Willey \& Cocilovo, 2007). The Gail Model has a limited use in this setting because it may underestimate the risk of breast cancer in someone who has a strong family history of breast and ovarian cancer, but has no affected first degree relatives (Willey et al., 2007).

Genetic health assessment tools are essential in the identification of individuals and their related family members who may be at high risk for cancer. Hence, it is necessary for nurses to determine if a client qualifies for a referral to hereditary counselling for possible genetic testing. This process begins with a meticulous nursing health assessment, with particular attention to a client's breast health history and a family cancer health history.

\section{Personal breast health history assessment}

The key components of a personal health history include nonmodifiable risk factors, such as a history of malignancies and/or benign breast pathologies, gender, age and a reproductive, and menstrual history. Modifiable factors include the use of oral contraception (OC), hormone replacement therapy (HRT), cigarette smoking, alcohol intake, obesity, and physical activity. A breast health history also assesses environmental factors, such as exposure to radiation or known carcinogenic chemicals.

A history of malignant or benign pathology impacts the risk profiles for breast cancer. A hallmark for a genetic referral is early onset breast cancer (i.e., before age 50), which has a fivefold increase of risk, while a personal history of endometrial or ovarian cancer carries a twofold risk increase (Willey et al., 2007). A combination of the early onset breast or ovarian (at any age) and/or bilateral (or multifocal) disease also heighten risk profiles (Petrucelli, Daly, Culver, \& Feldman, 2007). Several studies have revealed that elevated profiles for potential breast carcinoma include benign breast histology findings such as lobular carcinoma in situ, atypical lobular hyperplasia, atypical ductal hyperplasia and atypia (Brown, Moglia, \& Grumet, 2007).

Hormones influence cell growth. Hence, the changing levels of estrogen and progesterone in the female breast tissue increase the vulnerability to breast cancer in women versus men (Willey et al., 2007). Specific to age, the BRCA1 mutation carrier's breast cancer generally emerges in women less than 40, whereas for BRCA2 carriers, the frequency increases after the age of 50 (Nkondjock \& Ghadirian, 2004).
Rosen, Fan, and Isaacs (2005) contend that for BRCA mutation carriers, early age of first full-term pregnancy, increased parity, and late onset menarche have a protective effect against breast cancer. In a more recent study, Kotsopoulos and associates (2007) claim that the protective influences of early age of first full-term pregnancy are not seen with BRCA mutation carriers and, although multiparity confers protection for germline carriers of BRCA1, this protective effect does not apply to carriers of BRCA2 in which the underlying etiology is still not fully understood.

Over the years, although the use of exogenous hormones in the BRCA mutation carrier population has been studied extensively, the findings have been inconsistent. The National Hereditary Cancer Task Force (NHCTF) suggests caution regarding the recommendation of OC use in known BRCA mutation carriers aged 35 and older due to the potential increased risk of breast cancer. Studies on HRT and carriers of BRCA mutation have only been conducted in women who have had prophylactic bilateral salpingo-oophorectomy (BSO). The NHCTF supports the use of HRT (estrogen only) for short-term management of menopause symptoms, if non-hormonal regimens have failed to control symptoms (Horsman, 2007).

The evidence related to BRCA and smoking is also inconsistent. For example, while Cuningham (2008) recently reported a linear increase in the rates of breast cancer among mutation carriers who smoke cigarettes, this finding countered previous documentations of no association between these variables. The findings of McGuire et al. (2006) support previous research outcomes of no increased risk associated with alcohol and BRCA mutation carriers under the age of 50. The research literature also consistently reflects a delay in the onset of breast cancer for mutation carriers who are physically active and maintain a healthy body weight (Ronckers, Erdmann, \& Land, 2005). The research findings related to the associated risk of breast cancer for BRCA mutation carriers post ionizing radiation exposure are inconclusive. However, the literature does support an increased risk for anyone who has had radiation exposure prior to the age of 30 (Willey et al., 2007).

\section{Family oncology health history assessment}

A breast health assessment incorporates a family health history. Ideally, this history evaluates three generations on both sides of the family, with attention to the number of affected versus non-affected individuals, type of cancer, age at diagnosis, cancer presence in successive generations, and degree of relations to each other. In addition, information related to gender, ethnicity, cancer management regimen, and present health status (i.e., alive and well or date of death) should be elicited (Barse, 2006). Petrucelli et al. (2007) recommends risk assessments should also take into consideration a family history of a number of other cancer types and cancer syndromes that are associated with an increased risk of breast cancer (See Table 1).

\section{Genetic testing}

The gold standard for assessing susceptibility to a BRCA gene mutation is genetic testing. Genetic testing process begins with pretest counselling and informed consent of an individual who has been identified as high risk for a mutation. A risk assessment score that indicates $>10 \%$ probability of having a predisposition for a mutation satisfies most criteria standards for testing (Carter, 2001). Genetic testing criteria standards are also influenced by clinical reasoning based on personal and family risk factors, the impact of genetic test results on treatment management plans (i.e., surgical versus chemoprevention options), and the client's identified concerns and preferences (Berliner et al., 2007). Family members of an individual who has received a positive BRCA test outcome are also eligible for predictive genetic testing (Ensenauer, Michels, \& Reinke, 2005).

Testing generally involves extracting DNA from a peripheral blood sample and performing a comprehensive sequence analysis and/or target mutation analysis on the DNA (Petrucelli et al., 2007). Accuracy variations are seen among testing laboratories. Hence, the sensitivity 
of a full sequence analysis ranges from $80 \%$ to $95 \%$, and target mutation analysis is approximately $99 \%$ accurate (Sifri, Gangadharappa, \& Acheson, 2004). Brown et al. (2007) summarize test outcomes as positive, negative, or a variant of uncertain significance. Since testing methods are not $100 \%$ sensitive, negative results must be interpreted within the clinical context of the case at hand and the family history assessment (Ensenauer et al., 2005). There are a number of possible explanations for negative findings, including: it may be a sporadic cancer or the client may, in fact, be carrying a BRCA mutation that was not identified in the analysis, or may carry a mutation in another cancer susceptibility gene (Evans, Skrzynia, Susswein, \& Harlan, 2005). Alternatively, the report may confirm a variant of uncertain clinical significance, which means an indeterminate test outcome shows detectable changes in the DNA sequence; however, there are no clear indications if the changes will or will not affect the function of BRCA genes (Brown et al., 2007). A positive result confirms the presence of a mutation in the gene sequence, which places an individual at greater risk for developing breast cancer. However, it is not indicative of a definite future breast cancer. Hence, interpretation and disclosure of test results is complex and sensitive. Therefore, discussions are initiated by medical geneticists, genetic counsellors and advanced practice nurses with specialized training in genetics.

\section{Experiential perspective}

Mitchell et al. (1991) describe experiential responses as a selfreport of the examination of personal thoughts and feelings, which are affected by an individual's knowledge and meaning of the experience. These states are also influenced by one's cultural and social meaning of the experience. Based on a recent Cochrane Review, Sivell, Iredale, Gray, and Coles (2007) note that the psychological effects of genetic counselling and testing have not been extensively documented in the literature. However, existing reports describe the clients who seek genetic testing as healthy women who are at an increased risk and women who have a diagnosis of breast cancer. Their motive for testing is to acquire clarification about their own perceptions of highrisk status in order to make informed decisions about preventative strategies or medical management options. While women tend to seek testing as an action towards minimizing family risk, they do identify concerns about potential health insurance and work discrimination issues (Phelps, Wood, Bennett, Brain, \& Gray, 2007).

Phelps et al. (2007) assessed free text data questionnaires of women who were referred for breast genetic counselling and elected to

\section{Table 1. Hallmark indicators of increased}

hereditary susceptibility for BRCA1 and BRCA2

\section{Personal History}

Young age of onset $(<50$ years of age)

Bilateral cancer in paired organs

Male breast cancer

Multiple tumours in the same individual

\section{Successive generations affected}

Cancer in two or more first or second degree relatives

Young age of onset $(<50$ years of age)

Male breast cancer

Cancer types: Breast, ovarian, fallopian tube, prostrate, pancreatic, larynx, esophagus, stomach, gallbladder, bile duct, hematopoietic system, melanoma

Cancer Syndromes: Li-Fraumeni Syndrome, Cowden's Syndrome, Peutz-Jegher Syndrome, Hereditary Diffuse Gastric Cancer, Ataxia-Telangiectasia, Bloom Syndrome, Werner Syndrome, Xeroderma Pigmentosum

Ethnicity: Ashkenazi Jewish, Dutch, Icelandic

Adapted from Petrucelli et al. (2007) enrol in a psychological intervention trial $(\mathrm{N}=97)$. The analysis revealed that women were concerned about how they would cope if deemed to be at increased risk and how they would relay this information to their families. In addition, Phelps et al. (2007) found that adjustments to risk outcomes relied on individual perception of how well the risk assessment process met their expectations. Schlich-Bakker, ten Kroode, and Ausems, (2006), and Smith et al. (2008) study outcomes were similar to other studies, which supports the notion that the genetic testing process does not result in long-term psychological distress. Smith and associates (2008) noted no significant differences between levels of distress, perceived risk, and quality of life in women who attended a genetic testing clinic. They found that the women who declined genetic testing and those who received a positive test result reported increased distress at three months compared to their baseline overall distress scores, based on the Global Severity Index. It is of interest to note that for the women with positive BRCA status, distress scores peaked at three months following results notification and then returned to normal at six months. The clinical-based study by Schwartz, Peshkin, Hughes, Main, Isaacs, and Lerman (2002) also supported no increase in distress or perceived risk for women at six months after disclosure of a BRCA mutation status. In addition, the findings of Schwartz et al. (2002) indicated no heightened awareness in distress or perceived risk for relatives of the proband (i.e., the family member first tested) who had tested positive for a BRCA deleterious mutation.

\section{Nursing implications}

The HRTI Model provides a comprehensive framework for clinicians to evaluate individual needs based on the physiological, pathophysiological, behavioural, and experiential perspectives. The review of the literature, based on these perspectives, provides insight for clinical practice, education, and research. The role of the clinic oncology nurse in supporting clients who are going through genetic testing is multifaceted and encompasses interactions both pre- and post-genetic counselling.

Measurement tools, such as genetic history assessment forms, are critical for the identification of appropriate candidates for genetic counselling (Barse, 2006). Clinic oncology nurses are in the ideal position to inform clients of genetic services and to engage in discussions about the process. Preparatory discussion should touch on the roles of additional team members (i.e., geneticists and genetic counsellors), the benefits and limitations of genetic testing, timeframes associated with testing, and all plausible test outcomes. Throughout the process, clinic oncology nurses should continue to dialogue with clients regarding their identified needs and expectations of the genetic process. As well, it is important to offer supportive counselling and individualized education that is tailored to the client's decision and specific needs. Nurses must be astute to the potential need for psychosocial oncology referrals throughout the process, with the awareness of the possible heightened threshold around three months after the initial genetic consult (Smith et al., 2008). After clients have received genetic test results, breast clinic oncology nurses can reinforce this information, along with education regarding health, surveillance and management options (Ensenauer et al., 2005). Consequently, an understanding of the interpretation of genetic test results is imperative for nurses working in this speciality area. True negative test results indicate that the individual's risks for a breast cancer are comparable to the general population (Horsman, 2007). Nursing education for these clients should include age-appropriate breast screening, modifiable riskreduction strategies and information on prevention clinical trials. A finding of a variant of uncertain clinical significance, in light of a moderate to high-risk clinical picture, necessitates collaborative discussions between the client and genetic specialists, nursing, and various members of the oncology medical team.

Education for clients after a positive test result should include individualized counselling and education regarding age-appropriate risk for developing a breast or other gene-related cancers, surveillance strategies, preventative surgical and medical management options, potential clinical trials, implication for family members, 
and options regarding family information disclosure, as well as ongoing supportive counselling with appropriate multidisciplinary referrals (Sivell et al., 2007; Phelps et al., 2007).

Advances in BRCA genetic testing have created challenges for oncology nurses in the clinical setting. To gain a better understanding of the physiologic, pathophysiologic, behavioural, and experiential perspectives, it is essential for nurses to actively seek out educational opportunities, such as reviewing the related research literature, attending genetic conferences, and completing formal education in genetic oncology. This review of the literature suggests that further research is needed to establish evidence-based recommendations regarding the use of OCs with BRCA mutation carriers, HRT with carriers who have not had a BSO, and the risk for a breast cancer for carriers who have been exposed to ionizing radiation (Horsman, 2007; Willey et al., 2007). Sivell et al. (2007) and Smith et al. (2008) study findings highlight the need for ongoing research related to nursing interventions for the psy-

\section{References}

Barse, P.M. (2006). How to Perform a Genetic Assessment. In A.S. Tranin, A. Masny \& J. Jenkins (Eds.), Genetics in Oncology Practice: Cancer Risk Assessment (2nd ed., pp. 57-73). Pittsburgh, PA: Oncology Nursing Association.

Berliner, J.L., \& Fay, A.M. (2007). Risk assessment and genetic counseling for hereditary breast and ovarian cancer: Recommendations of the National Society of Genetic Counselors. The American Journal of Human Genetics, 16, 241-260.

Brown, K.L., Moglia, D.M., \& Grumet, S. (2007). Genetic counseling for breast cancer risk: General concepts, challenging themes and future directions. Breast Disease, 27, 69-96.

Canadian Cancer Society/National Cancer Institute of Canada. (2009). Canadian Cancer Statistics, ISSN 0835-2976.

Carroll, J.C., Cremin, C., Allanson, J., Blaine, S.M., Dorman, H., Gibbons, C.A., et al. (2008). Hereditary breast and ovarian cancers. Official Publication of the College of Family Physicians of Canada, 54(12), 1691-1692.

Carter, R.F. (2001). BRCA1, BRCA2 and breast cancer: A concise clinical review. Clinical and Investigative Medicine, 24(3), 147-157.

Chapman, D.D. (2007). Cancer genetics. Seminars in Oncology Nursing, 23(1), 2-9.

Cuningham, K. (2008). Smoking and risk of breast cancer in carriers of mutations in BRCA1 or BRCA2 aged less than 50 years. Breast Cancer Research and Treatment, 109, 67-75.

Ensenauer, R.E., Michels, V.V., \& Reinke, S.S. (2005). Genetic testing: practical, ethical, and counseling considerations. Mayo Clinical Proceedings, 80(1), 63-73.

Evans, J.P., Skrzynia, C., Susswein, L., \& Harlan, M. (2005). Genetics and the young woman with breast cancer. Breast Diseases, 23, 17-29.

Fackenthal, J.D., \& Olopade, O.I. (2007). Breast cancer risk associated with BRCA1 and BRCA2 in diverse populations. Nat Rev Cancer, 7(12), 937-48.

Horsman, D., Wilson, B.J., Avard, D., Meschino, W.S., Sing, C.K., Plante, M., et al. On behalf of the National Hereditary Cancer Task Force. (2007). Clinical management recommendations for surveillance and risk-reduction strategies for hereditary breast and ovarian cancer among individuals carrying a deleterious BRCA1 or BRCA2 mutation. Journal of Obstetrics and Gynaecology Canada, 29(1), 45-60.

Kotsopoulos, J., Lubinski, J., Lynch, H. T., Klijn, J., Ghadirian, P., \& Neuhausen, S.L., et al. The Hereditary Breast Cancer Clinical Study Group. (2007). Age at first birth and the risk of breast cancer in BRCA1 and BRCA2 mutation carriers. Breast Cancer Research and Treatment, 105(2), 221-228.

Loescher, L.J., \& Whitesell, L. (2006). The Biology of Cancer. In A.S. Tranin, A. Masny \& J. Jenkins (Eds.), Genetics in oncology practice: Cancer risk assessment (2nd ed., pp. 23-55). Pittsburgh, PA: Oncology Nursing Association. chological assessment of clients who are undergoing genetic counselling and testing, with particular attention to those who have declined genetic testing. The review has also identified a need for research studies assessing the implementation and evaluation of decision aids pre and post genetic counselling for the high-risk breast cancer population in an oncology nursing clinical practice setting.

\section{Conclusion}

The potential or actual diagnosis of a hereditary breast cancer may impact on numerous aspects of an individual, as well as family member's well being. The HRTI Model offers oncology nurse clinicians with a framework for understanding the biology of hereditary breast cancer, and the structure for obtaining a detailed personal and family oncology history assessment. This information can be utilized for individualized client education and supportive care. Thus, the model provides an excellent framework for oncology nurses to facilitate a holistic model of care.

McGuire, V., John, E.M., Felberg, A., Haile, R.W., Boyd, N.F., \& Thomas, D.C., et al. (2006). No increased risk of breast cancer associated with alcohol consumption among carriers of BRCA1 and BRCA2 mutations ages $<50$ years. Cancer Epidemiology, Biomakers \& Prevention, 15, 1565-1567.

Mitchell, P.H., Gallucci, B., \& Fought, S.G. (1991). Perspectives on human response to health and illness. Nursing Outlook, 39(4), 154-157.

Narod, S.A., \& Foulkes, W.D. (2004). BRCA1 and BRCA2: 1994 and beyond. Nature Reviews Cancer, September(4), 665-676.

Nkondjock, A., \& Ghadirian, P. (2004). Epidemiology of breast cancer among BRCA mutation carriers: An overview. Cancer Letters, 205, $1-8$.

Petrucelli, N., Daly, M.B., Culver, J.O., Feldman, G.L. (2007). BRCA1 and BRCA2 hereditary breast/ovarian cancer. Gene Reviews. Retrieved from http://www.ncbi.nlm.nih.gov/bookshelf/br.fcgi? book=gene\&part=brcal

Phelps, C., Wood, F., Bennett, P., Brain, K., \& Gray, J. (2007). Knowledge and expectations of women undergoing cancer genetic risk assessment: A qualitative analysis of free-test questionnaire comments. Journal of Genetic Counseling, 16(4), 505-514.

Ronckers, C.M., Erdmann, C.A., \& Land, C.E. (2005). Radiation and breast cancer: A review of current evidence. Breast Cancer Research, 7(1), 21-32.

Rosen, E.M., Fan, S., \& Isaacs, C. (2005). Review BRCA1 in hormonal carcinogenesis; basic and clinical research. Endocrine-Related Cancer, 12(3), 533-548.

Schlich-Bakker, K.J., ten Kroode, H.F.J., \& Ausems, M.G.E.M. (2006). A literature review of the psychological impact of genetic testing on breast cancer patients. Patient Education and Counseling, 62(1), 13-20.

Schwartz, M.D., Peshkin, B.N., Hughes, C., Main, D., Isaacs, C., \& Lerman. C. (2002). Impact of BRCA1/BRCA2 mutation testing on psychologic distress in a clinic-based sample. Journal of Clinical Oncology, 20(2), 514-520.

Sifri, R., Gangadharappa, S., \& Acheson, L.S. (2004). Identifying and testing for hereditary susceptibility to common cancers. A Cancer Journal for Clinicians, 54, 309-326.

Sivell, S., Iredale, R., Gray, J., \& Coles, B. (2007). Cancer genetics risk assessment for individuals at risk of familial breast cancer (Review). Cochrane Database of Systematic Reviews, (2), CD003721, 1-30.

Smith, A.W., Dougall, A.L., Posluszny, D.M., Somers, T.J., Rubinstein, W.S., \& Baum, A. (2008). Psychological distress and quality of life associated with genetic testing for breast cancer risk. Journal of Psychosocial Oncology, 17, 767-773.

Willey, S.C., \& Cocilovo, C. (2007). Screening and follow-up of the patient at high risk for breast cancer. Obstetrics and Gynecology, 110(6), 1404-1416.

Yoshida, K., \& Miki, Y. (2004). Role of BRCA1 and BRCA2 as regulators of DNA repair, transcription and cell cycle in response to DNA damage. Cancer Science, 95(11), 866-871. 